\title{
Conical diffraction and the dispersion surface of hyperbolic metamaterials
}

\author{
K. E. Ballantine, J. F. Donegan, and P. R. Eastham \\ School of Physics and CRANN, Trinity College Dublin, Dublin 2, Ireland
}

(Received 25 September 2013; revised manuscript received 6 May 2014; published 3 July 2014)

\begin{abstract}
Hyperbolic metamaterials are materials in which at least one principal dielectric constant is negative. We describe the refractive index surface, and the resulting refraction effects, for a biaxial hyperbolic metamaterial, with principal dielectric constants $\epsilon_{1}<0,0<\epsilon_{2} \neq \epsilon_{3}$. In this general case the two sheets of the index surface intersect, forming conical singularities. We derive the ray description of conical refraction in these materials and show that it is topologically and quantitatively distinct from conical refraction in a conventional biaxial material. We also develop a wave optics description, which allows us to obtain the diffraction patterns formed from arbitrary beams incident close to the optic axis. The resulting patterns lack circular symmetry and hence are qualitatively different from those obtained in conventional, positive index materials.
\end{abstract}

DOI: 10.1103/PhysRevA.90.013803

PACS number(s): 42.25.Bs, 42.25.Lc, 78.20.Ci, 78.67.Pt

\section{INTRODUCTION}

Hyperbolic metamaterials (HMMs), materials which have a negative dielectric constant in at least one direction, are attracting attention due to their interesting physics and myriad applications. They can be manufactured relatively simply from alternating layers of metal and dielectric, or by embedding metal rods in a dielectric background [1,2]. HMMs have recently been shown to have unique properties, described by effective medium theory [3], including a broadband infinite density of states [4], arbitrarily large values of the wave vector [5], and negative refraction [2,6]. This has led to many proposed applications, from imaging [7,8], sensing [9], and wave guiding $[10,11]$ to information processing [12].

The most common HMMs considered are uniaxial materials for which $\epsilon_{1}<0<\epsilon_{2}=\epsilon_{3}$ where $\epsilon_{i}$ are the principal dielectric constants. This leads to a hyperboloid isofrequency surface (refractive index surface) for the extraordinary ray. The change in topology from an ellipsoid to a hyperboloid is responsible for many of the important properties of these materials [13]. The general case, however, is a biaxial HMM, where $\epsilon_{1}<0<\epsilon_{2}<\epsilon_{3}$. Such a material could be realized as layers of metal and dielectric, where the dielectric material has uniaxial isotropy in the plane, or as rods of metal embedded in a dielectric with different rod spacings in the $x$ and $y$ directions [14]. The isofrequency surface for the extraordinary ray is then an asymmetric hyperboloid [15].

In this paper we present the full two-sheeted isofrequency surface of a HMM, which describes the propagation of both the ordinary and the extraordinary rays with orthogonal polarizations, and show that it contains conical singularities. These singularities are degenerate points where the two sheets intersect at a point in $k$ space. Similar conical singularities occur in conventional biaxial materials, i.e., $0<\epsilon_{1}<\epsilon_{2}<$ $\epsilon_{3}[16,17]$, and lead to the phenomenon of conical refraction, in which a beam of light is refracted into two concentric hollow cones $[17,18]$. We describe these intersections in the case of a HMM and derive a geometrical optics description of refraction for rays with wave vector close to the degeneracy, including establishing the polarization and the Poynting vector, or energy flow. This predicts refraction into two intersecting rather than concentric cones, an effect topologically distinct from that in a conventional biaxial crystal and completely lacking from a uniaxial HMM. We then extend this theory to develop a paraxial wave optics description of the propagation of light through these materials. This allows us to calculate the diffraction patterns formed from arbitrary beams incident on a biaxial HMM close to the optic axis. We find these patterns to be qualitatively different from those obtained in positive index materials, in particular lacking circular symmetry.

These conical singularities are, in some respects, similar to the Dirac points [19] that are of growing importance in solid-state physics. These points, where bands cross linearly at a particular frequency and wave vector, are best known in graphene $[20,21]$. Graphene has attracted huge theoretical and applied interest [22-24], with many new features attributable to the linear dispersion near a Dirac point, which means that the low-energy excitations are massless chiral Dirac fermions [21]. They thus provide a model of quantum electrodynamics with the limiting speed given by the Fermi velocity rather than the speed of light $[21,25]$. They also lead to effects such as the anomalous integer quantum Hall effect [26,27] and mean that electrons are immune to localization, propagating over large distances without scattering $[20,28]$. Tilted Dirac cones, which are not circularly symmetric around the degenerate wave vector, are similar to the skewed-cone intersections reported here and have previously been predicted in mechanically deformed graphene [29]. Dirac points in optical systems have been found in photonic crystals, as a result of the same lattice symmetry [30,31], or in materials with a frequency dependent permittivity, which may pass through zero at a particular frequency, leading to a degeneracy [32-34].

In these cases, however, a degeneracy occurs at a particular frequency, due to fine tuning the frequency to match the sublattice periodicity, or to match a zero of the frequency dependent dielectric constant. At other nearby frequencies there is generally no singularity. In contrast, biaxial materials have conical singularities in the isofrequency surface in $k$ space, which is directly comparable to a Fermi surface. The presence of these singularities depends on the symmetry of orthogonal polarizations in a crystal and does not rely on fine tuning of any parameter. In particular, we show that they occur within effective medium theory and argue that their presence is required on topological grounds. Since this implies that they occur over a finite range of frequencies they correspond to line, rather than point, degeneracies in 
the dispersion relation (which describes a three-dimensional surface in the four-dimensional space of $\omega$ and $\mathbf{k}$ ).

The remainder of this article is structured as follows. In Sec. II we describe the two-sheeted dispersion surface in a biaxial HMM and compare it to the case of positive $\epsilon$. In Sec. III we derive the ray optics description of refraction, for incident rays with initial wave vector close to the optic axis, in a biaxial HMM. In particular, we present the polarization and Poynting vector, i.e., the direction of energy flow, of the refracted rays. In Sec. IV we extend the theory to include small absorption in the material and show explicitly that the conical intersections persist. In Sec. V we develop a wave optics description of propagation near the optic axis of a biaxial HMM and present the diffraction pattern formed with a Gaussian input beam. In Sec. VI we discuss further the connection between conical singularities in optics and singularities in solid-state band structures. We make an explicit connection between the conical singularities described here and Dirac points by reformulating the diffraction theory in terms of the paraxial wave equation. Finally, in Sec. VII we summarize our conclusions.

\section{DISPERSION SURFACES}

We can describe a nanostructured metamaterial in the effective medium theory by a three-dimensional dielectric tensor $\epsilon_{i j}$ or by the principal dielectric constants, $\epsilon_{i}$, which are its components in the frame in which it is diagonal [35]. Effective medium theory describes the subwavelength patterning of different materials by an average anisotropic dielectric tensor according to the Maxwell-Garnett formulas [36]. Plane-wave solutions to Maxwell's equations in the medium lead to the Fresnel equation for the refractive index:

$$
\sum_{i} \frac{\epsilon_{i} \eta_{i}^{2}}{n^{2}-\epsilon_{i}}=0
$$

where $\eta$ is a unit vector in the direction of the wave vector k [35]. The two solutions for $n^{2}$ for a given direction $\eta$ form a two-sheeted dispersion surface [35], also known as an isofrequency surface or refractive index surface. At a fixed frequency, these surfaces give the phase velocity, or equivalently the wave vector magnitude, in the medium, for a given wave-vector direction. The ray or energy flow direction will be orthogonal to the dispersion surface at the point defined by that wave vector [37]. In the following we assume without loss of generality that $\epsilon_{1}<\epsilon_{2}<\epsilon_{3}$.

Figure 1 shows sections of the dispersion surfaces for a variety of materials. These surfaces are polar plots where the radial distance represents the refractive index experienced by a ray propagating in that direction in $k$ space. Equivalently, they are three-dimensional cuts of the full four-dimensional space of $\omega$ and $\mathbf{k}$, taken at a constant $\omega$. In the approximation where the dielectric constants depend weakly on frequency, these surfaces will simply contract or expand as $\omega$ is decreased or increased, respectively, meaning the critical points will trace out lines. Outside of this approximation the dispersion surface will change shape but the basic features will remain until the dielectric constants cross each other or zero. Hence, assuming a smooth dependence on frequency, there will always be a (a)

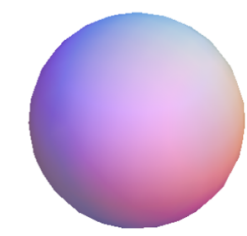

$0<\epsilon_{1}=\epsilon_{2}=\epsilon_{3}$

(d)

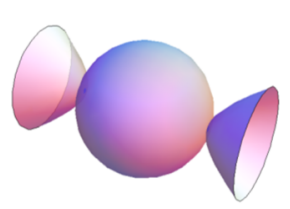

$\epsilon_{1}<0<\epsilon_{2}=\epsilon_{3}$

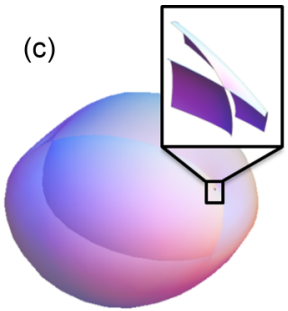

$0<\epsilon_{1}<\epsilon_{2}<\epsilon_{3}$ (b)

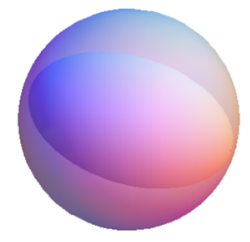

$0<\epsilon_{1}<\epsilon_{2}=\epsilon_{3}$ (e)

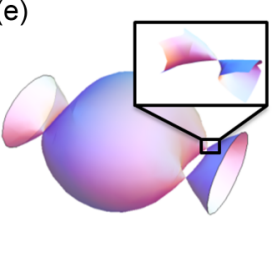

(f)

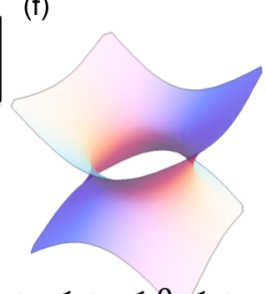

FIG. 1. (Color online) Isofrequency surfaces for various effective index materials: (a) isotropic, (b) uniaxial, (c) biaxial, (d) uniaxial-hyperbolic, (e) biaxial-hyperbolic type 1, and (f) biaxialhyperbolic type 2 . Shading is for perspective only. Additional cases not shown include $\epsilon_{1}=\epsilon_{2}<0<\epsilon_{3}$, which is identical to (f) but with circular cross sections, and $\epsilon_{3}<0$, in which case there are no real solutions. These surfaces are polar plots of the refractive index as a function of ray direction $\eta$. In the case of (b) and (d) the surfaces intersect at two points, at which they are parallel. In the case of (c) and (e) the surfaces have four conical intersections. Insets in (c) and (e) show cutaway closeups of the intersection points. Cuts through these intersections are presented in Fig. 2.

continuous range of frequencies for which these singularities exist.

The classical cases, $0<\epsilon_{i}$, are shown in the first row and are the subject of conventional crystal optics. The surfaces have positive curvature and finite area. The hyperbolic cases, $\epsilon_{1}<0$, shown in the second row, are the result of nanostructured materials which have properties not found in nature at optical frequencies. They have dispersion surfaces which are unbounded in $|k|$ at any frequency and feature both positive and negative curvature [1].

The possible classical materials fall into three categories. Figure 1(a) shows an isotropic material which has a single spherical dispersion surface. Once isotropy is broken, the surface splits into two as the two orthogonal polarizations experience different dielectric constants. For a uniaxial material, with two indices equal, these surfaces intersect at two points, along a single optic axis as shown in Fig. 1(b). However, the surfaces are parallel at the degenerate points, and so the normals remain well defined [38]. For a biaxial crystal, shown in Fig. 1(c), rotational symmetry is broken completely. The surfaces intersect at four points along two optic axes. The gradient of the surfaces is singular at the degenerate points and the normal is not well defined.

These singularities lead to the unique phenomenon of conical refraction [16]. For a general angle of incidence in an anisotropic medium, the two orthogonal polarizations of an incident ray are refracted into two rays with different 

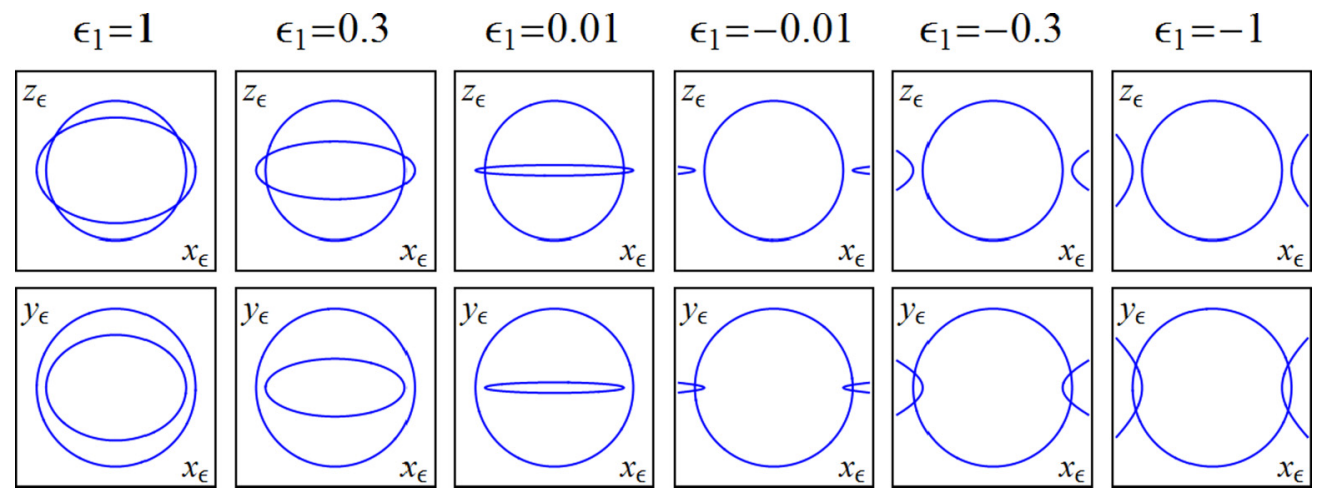

FIG. 2. (Color online) The transition from biaxial to biaxial-hyperbolic type 1 material as $\epsilon_{1}$ passes through zero. One of the dispersion surfaces changes topology from an ellipsoid to a hyperboloid. The intersection points move from the $x_{\epsilon}-z_{\epsilon}$ plane to the $x_{\epsilon}-y_{\epsilon}$ plane. The first row shows the surfaces in the $x_{\epsilon}-z_{\epsilon}$ plane $\left(y_{\epsilon}=0\right)$. The second row shows the surfaces in the $x_{\epsilon}-y_{\epsilon}$ plane $\left(z_{\epsilon}=0\right)$.

wave vectors, called the ordinary and extraordinary rays. In conical refraction, when the incident wave vector coincides with the optic axis, the two orthogonally polarized incident rays are refracted into two concentric cones which contain all polarizations at different points around each cone $[18,35]$.

When one of the dielectric constants becomes negative, leading to a hyperbolic metamaterial, there is a topological transition of one of the surfaces, from an ellipsoid to a hyperboloid. Figure 1(d) shows a uniaxial HMM. The surfaces again intersect at two points where they are parallel. In the case of a biaxial HMM, shown in Fig. 1(e), linear crossings occur. The hyperboloid and the ellipsoid intersect at four degenerate points. We describe for the first time these conical singularities in the dispersion surface of a biaxial HMM, and their associated refraction and diffraction effects. In the final case, where two of the three indices are negative, Fig. 1(f), there is again a single dispersion surface which is a type two hyperboloid [1] with no singularities. This single dispersion surface describes one polarization which can propagate in the material. For the orthogonal polarization the material is metallic, and absorbing, and hence there is no second real solution to the Fresnel equation.

In both Figs. 1(b) and 1(d) the two sheets have a quadratic degeneracy. Including the perturbation $\epsilon_{2} \neq \epsilon_{3}$ will clearly either open a gap or cause the quadratic intersection to split into two linear intersections, in line with general band theory. If a gap were to open, however, it would leave at least one closed surface which described the propagation of a different linear polarization at each point. The field of polarization directions described by this surface would form a tangential vector field on a closed two-dimensional surface. This is forbidden by the hairy ball theorem, unless the linear polarization vanishes at least once. Comparing with the Poincaré sphere representation for the polarization, we see that such points, if they occurred, would correspond to points with circular polarization. However, in the presence of chiral symmetry the two circular polarizations cannot have different refractive indices, so that there cannot be a gap at these points. Thus, in the presence of chiral symmetry, the existence of conical singularities in the isofrequency surface is required on topological grounds. In its absence, however, a gap does indeed appear [39].
The transition from a conventional biaxial material to a biaxial type $1 \mathrm{HMM}$ is shown in Fig. 2 as $\epsilon_{1}$ goes from positive to negative. As rotational symmetry in the $y_{\epsilon}-z_{\epsilon}$ plane is broken (note we use the subscript $\epsilon$ to denote the basis in which $\epsilon$ is diagonal), the degenerate points are free to move around the $x_{\epsilon}$ axis as $\epsilon_{1}$ varies. The points start in the $x_{\epsilon}-z_{\epsilon}$ plane and move closer to the $x_{\epsilon}$ axis as $\epsilon_{1} \rightarrow 0$. Then as the topological transition occurs the critical points change direction and move away from the $x_{\epsilon}$ axis into the $x_{\epsilon}-y_{\epsilon}$ plane.

The topological transition between the conical singularities of positive and negative index materials can be seen by calculating the solutions to the Fresnel equation, Eq. (1), which are degenerate. We find two sets of solutions:

$$
\begin{aligned}
& \eta_{1}= \pm \sqrt{\frac{\epsilon_{3}\left(\epsilon_{2}-\epsilon_{1}\right)}{\epsilon_{2}\left(\epsilon_{3}-\epsilon_{1}\right)}}, \\
& \eta_{2}=0 \\
& \eta_{3}= \pm \sqrt{\frac{\epsilon_{1}\left(\epsilon_{3}-\epsilon_{2}\right)}{\epsilon_{2}\left(\epsilon_{3}-\epsilon_{1}\right)}}
\end{aligned}
$$

and

$$
\begin{aligned}
& \eta_{1}= \pm \sqrt{\frac{\epsilon_{2}\left(\epsilon_{3}-\epsilon_{1}\right)}{\epsilon_{3}\left(\epsilon_{2}-\epsilon_{1}\right)}}, \\
& \eta_{2}= \pm \sqrt{\frac{-\epsilon_{1}\left(\epsilon_{3}-\epsilon_{2}\right)}{\epsilon_{3}\left(\epsilon_{2}-\epsilon_{1}\right)}}, \\
& \eta_{3}=0 .
\end{aligned}
$$

The first solution Eq. (2) is real, and therefore physical, when all the $\epsilon_{i}$ are positive. As $\epsilon_{1}$ becomes negative, $\eta_{3}$ in Eq. (2) becomes imaginary. The second solution, Eq. (3), then becomes the real, physically relevant, $\eta$. In this way the transition through $\epsilon_{1}=0$ separates topologically distinct sets of degenerate solutions.

Figure 3 shows the cross sections of the dispersion surfaces at the degenerate points, in the case of a conventional biaxial crystal and a biaxial hyperbolic material. For a conventional material, both surfaces have similar curvatures. The normals to the surfaces close to the optic axis, i.e., the axis which passes through one of the degenerate points, are shown. These normals indicate the direction of refraction for rays which 
(a)

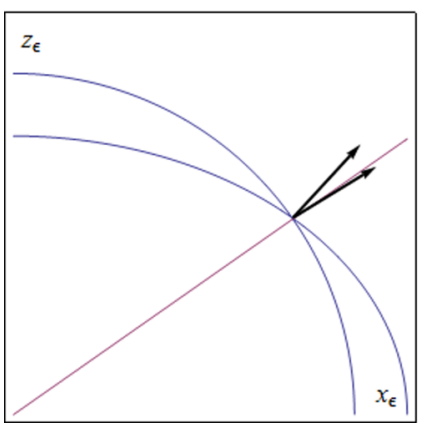

(b)

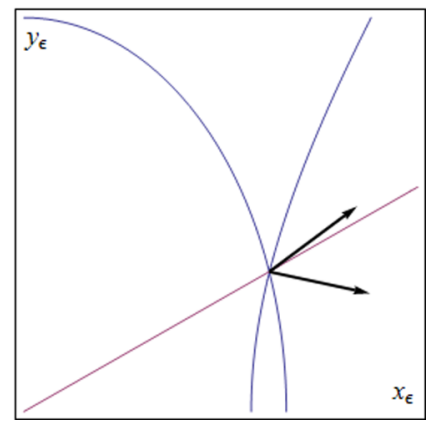

FIG. 3. (Color online) Cross sections of the isofrequency surfaces through the degenerate points for (a) a conventional biaxial material and (b) a hyperbolic biaxial material. The optic axis is shown by the straight line, and the approximate normals to the surfaces for a $\mathbf{k}$ vector passing close to this axis are shown by the arrows and are suggestive of the expected conical refraction. In the hyperbolic case the cone points toward rather than away from the $x_{\epsilon}$ axis.

approximately coincide with the optic axis. In the positive $\epsilon$ case, one points close to the optic axis while the other points away from the $x_{\epsilon}$ axis. In the case of a biaxial HMM the surfaces have opposite curvatures. This leads to one of the normals pointing towards the $x_{\epsilon}$ axis. When the full two-dimensional surface is considered, the normals shown here contribute to a cone which is skewed away from the optic axis, in a different direction in each case. In Fig. 3(b), one of the normals points downward, below the horizontal. If the material is cut so the interface is the $y_{\epsilon}-z_{\epsilon}$ plane, i.e., the normal is parallel to the $x_{\epsilon}$ axis, then this results in part of the cone being refracted back on the same side of the normal to the incoming ray, a phenomenon sometimes known as negative refraction. However, this term is also used to refer to negative phase velocity, which is not present in this case.

\section{GEOMETRICAL OPTICS}

We now turn to describing the refraction of light incident on a biaxial HMM, when the incident wave vector lies close to the optic axis, as shown in Fig. 3. To achieve this we calculate the refractive index surface experienced by the ray and the resulting Poynting vector of the refracted ray. We describe the ray by polar coordinates in a frame where the $x$ axis coincides with the optic axis, and the $z$ axis coincides with the $z_{\epsilon}$ axis, as illustrated in Fig. 4. $\theta$ is the angle between the ray and the optic axis, while $\phi$ is the azimuthal angle from the $y$ axis in the $y-z$ (transverse) plane. Expressing $\eta$ in terms of $\theta$ and $\phi$ and solving Eq. (1) we find the refractive index to first order in $\theta$ is

$$
n^{2}=\epsilon_{3}-\theta \epsilon_{\delta}(\cos \phi \pm 1)
$$

where

$$
\epsilon_{\delta}=\epsilon_{3} \sqrt{\frac{\left(\epsilon_{3}-\epsilon_{1}\right)\left(\epsilon_{2}-\epsilon_{3}\right)}{\epsilon_{1} \epsilon_{2}}}
$$

is a measure of the anisotropy of the medium. The surface described by Eq. (4) consists of two cones touching at their points, which is the linear approximation to the surface

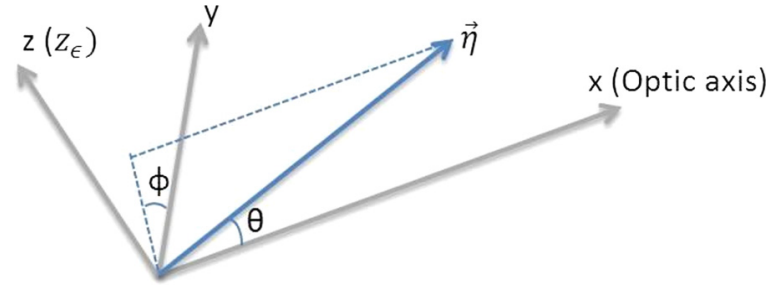

FIG. 4. (Color online) The coordinate system used to describe refraction near the optic axis in a biaxial HMM. The $x$ axis corresponds to the optic axis through the direction given by Eq. (3) while the $z$ axis corresponds to the $z_{\epsilon}$ axis. $\theta$ is the angular displacement of the ray from the optic axis while $\phi$ is the azimuthal angle of the ray in the transverse plane.

portrayed in Fig. 1(e) around one of the intersection points. Furthermore, we find the polarization of the two refracted rays is

$$
\frac{D_{z}}{D_{y}}=\frac{\sin \phi}{\cos \phi \pm 1}
$$

where $\mathbf{D}$ is the electric displacement field.

The results, Eqs. (4) and (6), describe the refractive index experienced by an incoming ray. A ray which comes from an azimuthal angle $\phi$ can be decomposed into the two orthogonal polarizations given by Eq. (6). These two polarizations experience the refractive indices given by Eq. (4). The polarizations are independent of $\theta$, as long as $\theta$ is small. Thus, for any ray not exactly coincident with the optic axis, there are two distinct polarization modes. As $\phi$ varies, the direction of polarization described by a given dispersion surface rotates, so that a ray with one linear polarization and azimuthal angle $\phi$ undergoes the same refraction as a ray with the orthogonal polarization and azimuthal angle $\phi+180^{\circ}$. However, Eq. (6) is undefined when $\theta=0$. Hence there is also a polarization degeneracy at the conical singularity where all polarizations experience the same refractive index.

Equation (4) differs from the usual case of conical refraction in a biaxial crystal in two noteworthy ways. First, $\epsilon_{3}$ plays the role of the average dielectric constant, despite being the largest of the three indices, while for a conventional biaxial crystal the median index $\epsilon_{2}$ plays this role. Second, the parameter $\epsilon_{\delta}$ depends on $\sqrt{\epsilon_{3}-\epsilon_{1}}$, which is a large parameter since $\epsilon_{1}$ is negative. In the conventional, $\epsilon_{i}>0$, case of conical refraction the corresponding form is $\epsilon_{\delta}=\epsilon_{2} \sqrt{\left(\epsilon_{2}-\epsilon_{1}\right)\left(\epsilon_{3}-\epsilon_{2}\right) / \epsilon_{1} \epsilon_{3}}$, which is usually small. The polarization modes given by Eq. (6) are identical to the positive $\epsilon$ case. Thus we do not expect the polarization profiles generated by conical refraction and diffraction to change.

We now calculate the Poynting vector using Eqs. (4) and (6) for the two orthogonal polarizations associated with each incident wave vector. The Poynting vector is, up to an overall constant, given by

$$
\mathbf{P}=\mathbf{E}^{*} \times \mathbf{H}
$$

$\mathbf{E}$ and $\mathbf{H}$ can be expressed in terms of $D_{z}$ and $D_{y}$, given by Eq. (6), using Maxwell's equations and the constitutive 

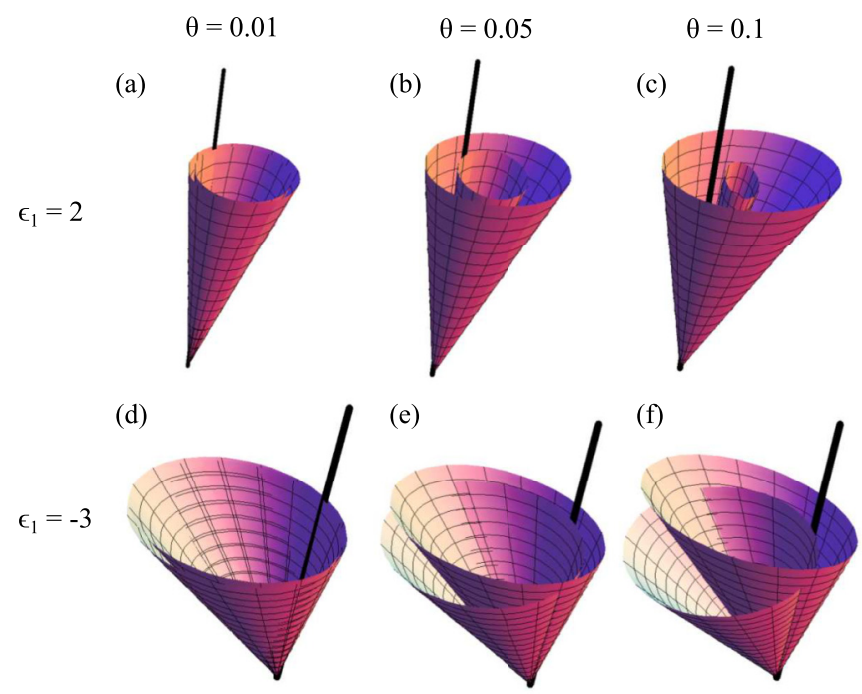

FIG. 5. (Color online) The loci of the Poynting vector of the two modes in a conventional biaxial material and a biaxial hyperbolic metamaterial, for wave vectors making angles $\theta$ and $\phi$ to the optic axis, as $\phi$ varies from zero to $2 \pi$. In the conventional case the cones are concentric, while in the hyperbolic case they intersect. For $\theta \rightarrow 0$ the cones are degenerate. As $\theta$ increases they move further apart. Parameters used are $\epsilon_{2}=3$ and $\epsilon_{3}=4$. Top row, $\epsilon_{1}=2$ : (a) $\theta=0.01$, (b) $\theta=0.05$, and (c) $\theta=0.1$. Bottom row, $\epsilon_{1}=-3$ : (d) $\theta=0.01$, (e) $\theta=0.05$, and (f) $\theta=0.1$. The solid black line indicates the optic axis, while the shading is for perspective only.

relations. The result,

$$
\begin{aligned}
P_{x}= & \frac{1}{\epsilon_{3}^{3 / 2}}+\theta \frac{\epsilon_{\delta}}{\epsilon_{3}^{5 / 2}}(\cos \phi \pm 1) \\
P_{y}= & \frac{\epsilon_{\delta}}{2 \epsilon_{3}^{5 / 2}}(1 \pm \cos \phi)+\frac{1}{\sqrt{\epsilon_{3}}} \theta\left[ \pm \frac{\epsilon_{\delta}^{2}}{4 \epsilon_{3}^{3}}(\cos \phi \pm 1)^{2}\right. \\
& \left.+\frac{1}{2}\left(\frac{1}{\epsilon_{1}}+\frac{1}{\epsilon_{2}}\right)(\cos \phi \pm 1) \mp \frac{1}{\epsilon_{3}}\right] \\
P_{z}= & \pm \frac{\epsilon_{\delta}}{2 \epsilon_{3}^{5 / 2}} \sin \phi+\frac{1}{\sqrt{\epsilon_{3}}} \theta\left[\frac{\epsilon_{\delta}^{2}}{4 \epsilon_{3}^{3}}(\cos \phi \pm 1) \sin \phi\right. \\
& \left.+\frac{1}{2}\left(\frac{1}{\epsilon_{1}}+\frac{1}{\epsilon_{2}}\right) \sin \phi\right]
\end{aligned}
$$

is compared with the $\epsilon_{i}>0$ case in Fig. 5 for three values of $\theta$.

Equations (6) and (8) together describe the refraction of an incoming ray with wave vector at a small angle $\theta$ to the optic axis and an azimuthal angle $\phi$ in the perpendicular plane. As $\phi$ is varied, the resulting rays sweep out two intersecting cones while the polarization component which is refracted into each cone also varies. For $\theta=0$ a single ray of any polarization is refracted into a complete cone, containing all polarizations. However, any realistic incoming beam will be a superposition of rays with the $\theta=0$ ray contributing an infinitesimal amount to the resulting pattern [18].

Figure 5 shows the loci of the Poynting vectors at different fixed angles $\theta$ as the azimuthal angle $\phi$ is varied, for a biaxial conventional material and a biaxial HMM. This is indicative of the paths taken by refracted rays in the material. The figures show that the usual result of two concentric cones [18] changes to the topologically distinct case of two intersecting cones. At $\theta \approx 0$ the cones are degenerate and skewed away from the optic axis. The degeneracy is clear from Eq. (8). For $\theta=0$ the terms which depend on $\phi$ take the same value for one mode at a given $\phi$ as for the other mode at $\phi+\pi$. As $\theta$ increases, the cones move in opposite directions along the $y$ axis, so that they intersect and for large enough $\theta$ will separate entirely. We note that this is due to a particular term in the Poynting vector, Eq. (8),

$$
P_{y} \propto \cdots+\theta\left[\frac{1}{2}\left(\frac{1}{\epsilon_{1}}+\frac{1}{\epsilon_{2}}\right)(\cos \phi \pm 1) \mp \frac{1}{\epsilon_{3}}\right],
$$

which is the dominant term for the movement of the cones as $\theta$ increases. For $\epsilon_{1} \approx-\epsilon_{2}$, the first term in Eq. (9) is small, and so the two modes have terms $\approx \mp \theta / \epsilon_{3}$ in $P_{y}$ of opposite sign with little dependence on $\phi$. This means the entire cones will move in opposite directions as $\theta$ increases. There is a corresponding term in the conventional case, but there if $\epsilon_{1} \approx \epsilon_{2} \approx \epsilon_{3}$ it is the constant terms $\pm 1 / 2 \epsilon_{1} \pm 1 / 2 \epsilon_{2} \mp 1 / \epsilon_{3}$ which approximately cancel, leaving a term which is dominated by $\cos \phi$. Thus the centers of the cones do not move in this case.

\section{ABSORPTION}

So far it has been assumed that although the permittivity may be negative it will always be real. Since hyperbolic metamaterials contain a large proportion of metal, they will always have some absorption, leading to an imaginary part of the effective permittivity. Although metals generally have high absorption, it is possible to design hyperbolic metamaterials with a small imaginary part of $\epsilon$ over a range of frequencies [3]. Nevertheless it is important to consider how losses will affect the basic theory. Previous figures have plotted the real solutions of the Fresnel equation. In directions in which only one real solution exists, the other solution is completely imaginary and thus evanescent. When the permittivity is complex, all solutions are complex and represent waves which travel with some absorption, which depends on the size of the imaginary component.

Figure 6 shows the isofrequency surface in the $x_{\epsilon}-y_{\epsilon}$ plane when each principal dielectric constant has an imaginary part of 0.3 . This corresponds to an isotropic absorption; anisotropic absorption does not qualitatively change the results. Note that an isotropic material with $\epsilon=2+0.3 i$ would have an imaginary refractive index of $\kappa=0.1$, meaning the decay length of the intensity $\lambda /(4 \pi \kappa)$ would be less than a wavelength. Hence the imaginary part we are considering is small but not negligible. We see from Fig. 6 that the crossings identified in the absence of absorption remain and are not destroyed by the introduction of a complex permittivity. Furthermore the wave vector at the crossings has a small imaginary component, relative to its real component, meaning that these crossings correspond to (mostly) propagating solutions with some absorption. The persistence of intersections is ensured by the topological argument given previously, as the absorption does not break the symmetry between left and right circular polarizations. 


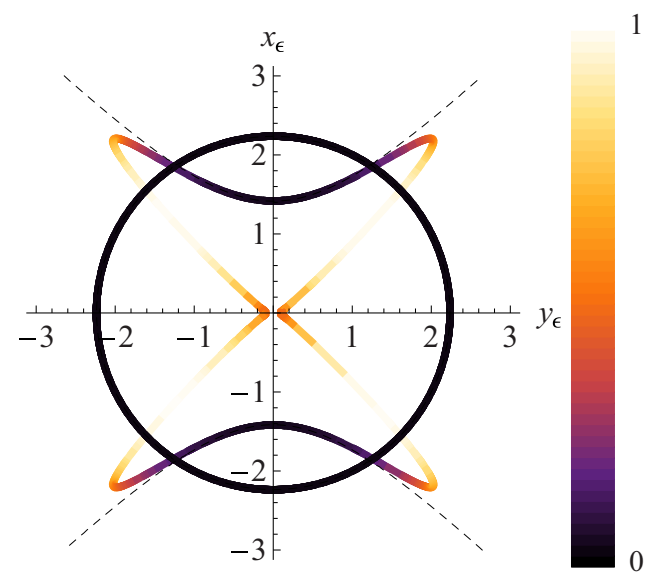

FIG. 6. (Color online) Isofrequency surface in the $x_{\epsilon}-y_{\epsilon}$ plane $\left(z_{\epsilon}=0\right)$ showing conical intersection in the presence of loss, with $\epsilon_{1}=-2+0.3 i, \epsilon_{2}=2+0.3 i$, and $\epsilon_{3}=5+0.3 i$, similar to the bottom right panel of Fig. 2. This is a polar plot of the real part of the refractive index with direction, with color representing the imaginary part of the refractive index, i.e., the absorption. White represents solutions with large absorption, and black represents those which are fully propagating. The original intersection remains a mostly propagating solution. An additional intersection appears which is mostly imaginary. The inclusion of an imaginary component to the effective medium theory is enough to prevent the dispersion surface becoming infinite. The dashed line shows the continuation of hyperbola in case of real $\epsilon$.

We also note, from Fig. 6, that in the case of complex dielectric constants the refractive index no longer goes to infinity: the open hyperboloid becomes closed and finite. This is purely a result of including losses, without leaving the effective medium theory. The hyperboloid dispersion surface bends back at finite $\mathbf{k}$, intersecting the ellipsoidal surface again. This second intersection has a large imaginary component, meaning that rays in this direction will decay quickly. These new intersections also occur in other directions of $\boldsymbol{\eta}$, where they are also mainly evanescent. As the imaginary component of $\epsilon$ is increased, this finite hyperboloid shape will decrease in size, until the mostly real and mostly imaginary intersections approach each other and finally disappear. However, mostly imaginary intersections also appear in the $x_{\epsilon}-z_{\epsilon}$ plane and remain for large imaginary components, in keeping with our previous topological argument.

\section{DIFFRACTION}

A complete treatment of optics near the conical singularities in a HMM must allow for diffraction of the incident and refracted beams. Here we develop such a treatment and obtain formulas for the diffraction patterns generated by arbitrary beams, incident on a biaxial HMM, with wave vectors close to the optic axis. We follow the method of [17]; in particular we use the angular spectrum representation to calculate the contribution of each input ray to the beam at a fixed propagation distance. Describing beams propagating close to the optic axis, which we will continue to label as the $x$ axis, the field at a position $x$ in the crystal consists of a sum of plane-wave components which pick up a phase on propagating:

$$
\begin{aligned}
E_{\text {out }}= & \iint d k_{y} d k_{z} E_{\text {in }}\left(k_{y}, k_{z}\right) \exp \left[i\left(k_{y} y+k_{z} z\right)\right] \\
& \times \exp \left(i x \sqrt{k_{T}^{2}-k_{y}^{2}-k_{z}^{2}}\right),
\end{aligned}
$$

where $E_{\text {in }}\left(k_{y}, k_{z}\right)$ is the two-dimensional Fourier transform of the input field in the plane $x=0$. However, the magnitude of the total wave vector in the crystal $k_{T}$ is $n k_{0}$, with $n$ depending on the direction of the ray, i.e., on $k_{y}$ and $k_{z}$. We can express the refractive index given by Eq. (4) in terms of the relative transverse momentum $\mathbf{p}=\boldsymbol{k}_{\perp} / k$, where $k=\sqrt{\epsilon_{3}} k_{0}$ is the magnitude of a wave vector lying directly along the optic axis. For small $\theta$ the transverse momenta are related to the angles defined in Fig. 4 by $p_{z}=\theta \sin (\phi), p_{y}=\theta \cos (\phi)$, and $p=|\mathbf{p}|=\theta$. The lowest-order terms, linear in $p$, lead to refraction into a simple cone which dominates the diffraction pattern. To reveal the fine structure we expand to second order, giving

$$
\begin{aligned}
n^{2} & \approx \epsilon_{3}-\epsilon_{\delta}\left(p_{y} \pm p\right)+\left(\epsilon_{\Delta} p \pm \frac{\epsilon_{\delta}^{2}}{\epsilon_{3}} p_{y}\right)\left(p \mp p_{y}\right) \\
& \equiv \epsilon_{3}\left[1+\mu\left(p_{y}, p\right)\right],
\end{aligned}
$$

where

$$
\epsilon_{\Delta}=\frac{\epsilon_{3}^{2}}{\epsilon_{1} \epsilon_{2}}\left(2 \epsilon_{3}-\epsilon_{1}-\epsilon_{2}\right)
$$

Letting $k_{T}^{2}=n^{2} k_{0}^{2}=k^{2}\left[1+\mu\left(p_{y}, p\right)\right]$ we can expand the square root in the final exponent of Eq. (10), again to $O\left(p^{2}\right)$, giving

$$
\begin{aligned}
\sqrt{k_{T}^{2}-k_{\perp}^{2}} & =\sqrt{n^{2} k_{0}^{2}-k^{2} p^{2}} \\
& =k \sqrt{1+\mu\left(p, p_{y}\right)-p^{2}} \\
& \approx k\left(1+\frac{1}{2} \mu\left(p, p_{y}\right)-\frac{1}{8} \mu\left(p, p_{y}\right)^{2}-\frac{1}{2} p^{2}\right),
\end{aligned}
$$

where we keep terms up to $O\left(p^{2}\right)$ in $\mu^{2}$.

The integral Eq. (10) with the approximation given in Eq. (13) gives the paraxial approximation to the electric field at a plane $x>0$, valid for small transverse momentum $p \ll 1$ or equivalently $k_{\perp} \ll k$. The term in the exponent proportional to $x p_{y}$ leads to a skew away from the optic axis in the cone, as suggested by Fig. 5, which can be included in the definition of a new transverse coordinate which follows the center of the cone $\mathbf{r}_{\perp}^{\prime}=\mathbf{r}_{\perp}+A x \hat{e}_{y}$ such that $\mathbf{p}$. $\mathbf{r}_{\perp}+A x p_{y}=\mathbf{p} \cdot\left(\mathbf{r}_{\perp}+A x \hat{e}_{y}\right)=\mathbf{p} \cdot \mathbf{r}_{\perp}^{\prime}$. The remaining terms which depend on $p_{y}$ cannot be absorbed in this way and lead to a noncircular asymmetry in the diffraction pattern. For simplicity we focus on the case of a circularly polarized, or unpolarized, input beam. For each wave vector the two orthogonal eigenpolarizations, given by Eq. (6), will then be present in equal amounts and will not interfere with each other. We consider a crystal of finite length $l<x$, so that the field propagates a length $l$ through the crystal before propagating a length $x-l$ in free space. Propagation beyond the crystal is described by an identical integral to Eq. (10) with the input field taken at the plane $x=l$ and with $k_{T}=k_{0}$. The intensity at 
a point $\left(x, \mathbf{r}_{\perp}^{\prime}\right)$ can then be written as the sum of the diffracted intensities from each eigenpolarization:

$$
I=\left|b_{+}\right|^{2}+\left|b_{-}\right|^{2} \text {. }
$$

Expressing Eq. (10) in terms of $p$ and using Eq. (13) gives

$$
\begin{aligned}
b_{ \pm}\left(x, \mathbf{r}_{\perp}^{\prime}\right)= & \frac{k}{2 \pi} e^{i k x} \iint d^{2} p a(\mathbf{p}) \exp \left(i k \mathbf{p} \cdot \mathbf{r}_{\perp}^{\prime}\right) \\
& \times \exp \left\{-i k p^{2}\left[\beta l+\frac{1}{2} \sqrt{\epsilon_{3}}(x-l)\right]\right\} \\
& \times \exp \left(-i k l \alpha p_{y}^{2}\right) \\
& \times \exp \left[ \pm i k l p\left(\gamma+\delta p_{y}\right)\right],
\end{aligned}
$$

where $a(\mathbf{p})$ is the Fourier transform of the input field and $\alpha, \beta, \gamma$, and $\delta$ are all expressed in terms of $\epsilon_{i}$; the explicit forms are given in the Appendix. These parameters control the diffraction patterns and have the following interpretations: $\beta$ is a propagation constant, $\gamma$ is proportional to the angle of the cone opening, and $\alpha$ and $\delta$ control the fine structure of the diffraction pattern leading to circular asymmetry.

As a specific application of the diffraction formulas, Eqs. (14) and (15), we show in Fig. 7 the conical diffraction pattern formed for a Gaussian beam, $a(p)=$ $k w^{2} \exp \left(-k^{2} p^{2} w^{2} / 2\right)$. The beam waist $w$ is taken as the unit length scale. The resulting intensity profile is plotted in the focal image plane, $x=l-2 \beta l / \sqrt{\epsilon_{3}}$, where the resulting ring structure is sharpest. This position corresponds to the image of the input beam waist in an isotropic crystal of index $\sqrt{\epsilon_{3}}$, and the pattern here can be imaged with a lens if it occurs inside or before the crystal [17]. As $\alpha, \beta, \gamma$, and $\delta$ all appear multiplied by $l$ for propagation inside the crystal; the length of the crystal is only important relative to the overall scale of these parameters; e.g., a short, strongly diffracting crystal will have the same effect as a long, weakly diffracting one. The parameter $\gamma l$ is chosen to give a ring radius $r_{0} \approx 50 \mathrm{w}$ to ensure well-developed rings while the other parameters are

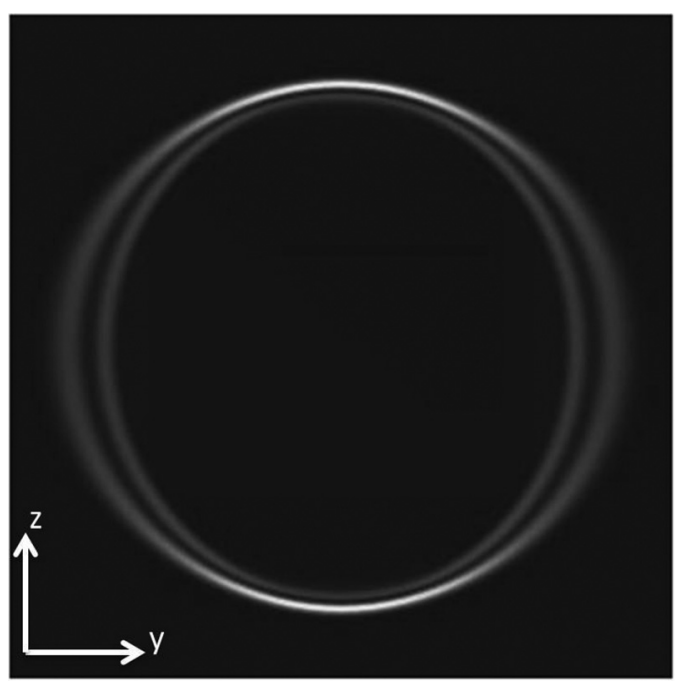

FIG. 7. The intensity profile formed by conical diffraction of a Gaussian beam in a hyperbolic metamaterial, in the focal image plane (see text). The pattern is generated from the paraxial diffraction integral, Eq. (15), with $\alpha l=10$ and $\delta=0$. $\alpha l=10, \delta l=0$. This choice allows us to show the asymmetry of the beam on the same scale as the overall conical refraction.

Like the positive $\epsilon$ case, the diffraction pattern consists of two rings. In contrast to that case, however, the diffraction pattern is not circularly symmetrical. The rings are broadened in the $y$ direction but remain tight in the $z$ direction. This is in agreement with Fig. 5, which shows the cones moving apart in the $y$ direction with increasing $p$. The diffraction pattern is bounded approximately on the inside and the outside by the arcs of two intersecting circles, also in agreement with the ray diagram. In addition there is a dark ring. This is purely an effect of diffraction and is not predicted by geometrical optics [18]. A similar dark ring, known as the Pogendorff ring, also appears in the conventional positive $\epsilon$ case.

\section{DISCUSSION}

As discussed in the introduction, a key feature of our results is the existence of linear intersections in the isofrequency surface in HMMs. These resemble the Dirac points that are of great interest in both condensed-matter physics and optics. It is therefore important to consider the relation between these phenomena carefully.

The dispersion surfaces describing the propagation of light in a biaxial material can be related to a band structure in two ways. The most straightforward is to consider the full dispersion relation $\omega(\mathbf{k})$ of light, which is a surface in the four-dimensional space of $\omega$ and $\mathbf{k}$, and compare it with the corresponding dispersion relation for electrons in a periodic lattice. In this case, the isofrequency surfaces described here are directly equivalent to a constant energy surface like the Fermi surface, and not directly to the dispersion relation as usually plotted. Both are, of course, cross sections of the full dispersion relation in the four-dimensional space of $\omega$ and $\mathbf{k}$, but in different directions.

For electrons there are two spin states related by time reversal, so that if time-reversal symmetry is present $\omega_{+}(\mathbf{k})=$ $\omega_{-}(-\mathbf{k})$. If there is spatial inversion symmetry then we furthermore have $\omega_{-}(\mathbf{k})=\omega_{-}(-\mathbf{k})$. Hence if these two symmetries are present there is only one, doubly degenerate, sheet to the Fermi surface. This is a case of Kramer's degeneracy. If one of these symmetries is broken then the spin-up and spin-down electrons can have different Fermi surfaces which may have conical intersections analogous to those described here, with the most common example being ferromagnetism [40].

For photons there are also two states, corresponding to the two polarizations, but these are related not by time-reversal symmetry, but by electromagnetic duality. This symmetry is present if the electric and magnetic fields can be interchanged. In most materials it is broken, because $\epsilon \neq \mu$, and this allows full frequency gaps to open, for example in a photonic crystal [41]. In terms of the isofrequency surfaces the (usual) breaking of this symmetry lifts the polarization degeneracy for most directions, leaving only the isolated point singularities described here.

There is, however, a less immediate but stronger connection between conical singularities and Dirac points, based on the well-known equivalence between the Schrodinger equation in two plus one dimensions and the paraxial Helmholtz equation. To demonstrate this connection in the present case, 
we construct the paraxial Helmholtz equation describing conical diffraction in a biaxial HMM. We begin by writing the electric field as a plane wave times a slowly varying envelope function:

$$
E(\mathbf{r})=A(\mathbf{r}) \exp (i k x),
$$

where $A(\mathbf{r})$ varies slowly with $x$. The diffracted field given by Eq. (15) can be expressed as the two-dimensional transverse input field evolving in the $x$ direction as

$$
E\left(\mathbf{r}_{\perp}, x\right)=\exp \left(-i k \int_{0}^{x} d x^{\prime} H\left(\mathbf{p}, x^{\prime}\right)\right) E\left(\mathbf{r}_{\perp}, 0\right),
$$

where for conical diffraction in a HMM we find that the Hamiltonian is

$$
H=\alpha p_{y}^{2}+\beta p^{2}+\left(\gamma+\delta p_{y}\right) \mathbf{s} \cdot \mathbf{p}
$$

for $x<l$ and is the free Hamiltonian $p^{2} / 2$ for $x>l$. Here $\mathbf{s}=$ $\left\{\sigma_{3}, \sigma_{1}\right\}$ is a vector of Pauli matrices in a Cartesian basis and $\mathbf{p}$ is formally represented by $-i \nabla_{\perp} / k$. The envelope function, thus, obeys the paraxial Helmholtz equation, which takes the form

$$
H A=\frac{i}{k} \frac{\partial A}{\partial x} .
$$

Since this is equivalent to the Schrödinger equation [42], the propagation with $x$ of the two-dimensional transverse beam is equivalent to the evolution with time of the wave function for a spin-1/2 particle. The birefringence of a biaxial material appears as a spin-orbit coupling, whose explicit form, close to the optic axis for a HMM, can be seen in Eq. (18). This form, with different definitions of the constants, also applies to a conventional biaxial material, but in that case the anisotropic terms proportional to $\alpha$ and $\delta$ are negligible and can be dropped [17].

Since light (of a fixed frequency) propagates in space according to Eq. (19), with $x$ playing the role of time, the propagation constant $k_{x}$ can be interpreted as the energy. The isofrequency surfaces can thus be seen as a dispersion relation, giving the propagation constant as a function of the two transverse momenta $k_{y}, k_{z}$. The point intersections in the isofrequency surfaces then correspond to Dirac points for twodimensional electrons; specifically, the point intersections discussed here are the Dirac points of the Hamiltonian, Eq. (18).

Dirac points in two-dimensional materials have been of interest for their role in topological insulators and topologically protected edge states $[43,44]$. In a hexagonal lattice such as graphene, subject to time-reversal symmetry and spatial inversion symmetry, the electronic band structure must contain Dirac points. These degeneracies can be lifted by breaking spatial inversion symmetry, leading to a trivial insulator, or by breaking time-reversal symmetry, leading to a topological insulator [45]. Hence, work on topological effects in photonic systems has focused on Dirac points, primarily in the full frequency dispersion $\omega(\mathbf{k})[41,46,47]$. More recently, however, attention has shifted to the analogous Dirac, or conical, intersections in the paraxial propagation constant surface $[39,48,49]$. Understanding the effects of different symmetries on these two dispersion surfaces could therefore help progress toward topologically protected photonic systems.

\section{CONCLUSIONS}

These results illustrate the unique singularities found in hyperbolic metamaterials when all three indices are allowed to vary independently. By examining the full dispersion surface of a general, biaxial, hyperbolic metamaterial, we have identified conical singularities at which the refraction direction is not defined. We have found the approximate dispersion surface and the refracted Poynting vector for a ray traveling close to the axis of these singularities. We have shown that this leads to a new form of refraction which does not appear in the usual uniaxial HMMs and is topologically and quantitatively different from the phenomenon of conical refraction which occurs in ordinary biaxial materials. These propagating solutions remain when a small imaginary component is included, leading to a small amount of absorption, with additional mostly evanescent singular solutions also appearing. We have also calculated the diffraction pattern for a beam traveling through such a material. We have found that the diffracted beam is generally not circularly symmetric and that, similar to the positive $\epsilon$ case, a dark ring appears where ray optics predicts the largest intensity.

\section{ACKNOWLEDGMENTS}

This work was supported by Science Foundation Ireland Grant No. SIRG I/1592 and by the Higher Education Authority under Programme for Research in Third-Level Institutions funding cycle 5. The authors wish to thank Prof. J. G. Lunney for useful discussions.

\section{APPENDIX}

We provide the parameters used in the diffraction theory in terms of the dielectric constants of the material:

$$
\begin{aligned}
& \alpha=\frac{\epsilon_{\delta}}{8}-\frac{\epsilon_{\Delta}^{2}}{2 \epsilon_{3}}, \quad \beta=\frac{1}{2}\left(\epsilon_{\Delta}-1\right)+\frac{1}{8} \epsilon_{\delta}, \\
& \gamma=\frac{1}{2} \epsilon_{\delta}, \quad \delta=\frac{\epsilon_{\delta}^{2}}{2 \epsilon_{3}}+\frac{\epsilon_{\delta}}{4}-\frac{\epsilon_{\Delta}}{2},
\end{aligned}
$$

recalling from Eqs. (5) and (12) that

$$
\begin{aligned}
& \epsilon_{\delta}=\epsilon_{3} \sqrt{\frac{\left(\epsilon_{3}-\epsilon_{1}\right)\left(\epsilon_{2}-\epsilon_{3}\right)}{\epsilon_{1} \epsilon_{2}},} \\
& \epsilon_{\Delta}=\frac{\epsilon_{3}^{2}}{\epsilon_{1} \epsilon_{2}}\left(2 \epsilon_{3}-\epsilon_{1}-\epsilon_{2}\right) .
\end{aligned}
$$

[1] C. L. Cortes, W. Newman, S. Molesky, and Z. Jacob, J. Opt. 14, 063001 (2012).
[2] V. A. Podolskiy and E. E. Narimanov, Phys. Rev. B 71, 201101 (2005). 
[3] O. Kidwai, S. V. Zhukovsky, and J. E. Sipe, Phys. Rev. A 85, 053842 (2012).

[4] Z. Jacob, I. I. Smolyaninov, and E. E. Narimanov, Appl. Phys. Lett. 100, 181105 (2012).

[5] X. Yang, J. Yao, J. Rho, X. Yin, and X. Zhang, Nat. Photon 6, 450 (2012).

[6] D. R. Smith, P. Kolinko, and D. Schurig, J. Opt. Soc. Am. B 21, 1032 (2004).

[7] Z. Jacob, L. V. Alekseyev, and E. Narimanov, Opt. Express 14, 8247 (2006).

[8] Z. Liu, H. Lee, Y. Xiong, C. Sun, and X. Zhang, Science 315, 1686 (2007).

[9] A. V. Kabashin, P. Evans, S. Pastkovsky, W. Hendren, G. A. Wurtz, R. Atkinson, R. Pollard, V. A. Podolskiy, and A. V. Zayats, Nat. Mater. 8, 867 (2009).

[10] A. A. Govyadinov and V. A. Podolskiy, Phys. Rev. B 73, 155108 (2006).

[11] Y. He, S. He, J. Gao, and X. Yang, J. Opt. Soc. Am. B 29, 2559 (2012).

[12] G. A. Wurtz, R. Pollard, W. Hendren, G. P. Wiederrecht, D. J. Gosztola, V. A. Podolskiy, and A. V. Zayats, Nat. Nano. 6, 107 (2011).

[13] H. N. S. Krishnamoorthy, Z. Jacob, E. Narimanov, I. Kretzschmar, and V. M. Menon, Science 336, 205 (2012).

[14] J. Elser, R. Wangberg, V. A. Podolskiy, and E. E. Narimanov, Appl. Phys. Lett. 89, 261102 (2006).

[15] J. Sun, J. Zeng, and N. M. Litchinitser, Opt. Express 21, 14975 (2013).

[16] M. V. Berry and M. R. Dennis, Proc. R. Soc. A 459, 1261 (2003).

[17] M. V. Berry, Journal of Optics A 6, 289 (2004).

[18] D. L. Portigal and E. Burstein, J. Opt. Soc. Am. 59, 1567 (1969).

[19] P. R. Wallace, Phys. Rev. 71, 622 (1947).

[20] K. S. Novoselov, A. K. Geim, S. V. Morozov, D. Jiang, Y. Zhang, S. V. Dubonos, I. V. Grigorieva, and A. A. Firsov, Science 306, 666 (2004).

[21] A. H. Castro Neto, F. Guinea, N. M. R. Peres, K. S. Novoselov, and A. K. Geim, Rev. Mod. Phys. 81, 109 (2009).

[22] A. K. Geim and K. S. Novoselov, Nat. Mater. 6, 183 (2007).

[23] D. R. Cooper, B. D’Anjou, N. Ghattamaneni, B. Harack, M. Hilke, A. Horth, N. Majlis, M. Massicotte, L. Vandsburger, E. Whiteway, and V. Yu, International Scholarly Research Notices 2012, 501686 (2012).

[24] S. Das Sarma, S. Adam, E. H. Hwang, and E. Rossi, Rev. Mod. Phys. 83, 407 (2011).

[25] K. S. Novoselov, A. K. Geim, S. V. Morozov, D. Jiang, M. I. Katsnelson, I. V. Grigorieva, S. V. Dubonos, and A. A. Firsov, Nature (London) 438, 197 (2005).
[26] V. P. Gusynin and S. G. Sharapov, Phys. Rev. Lett. 95, 146801 (2005).

[27] Y.Zhang, Y.-W. Tan, H. L. Stormer, and P. Kim, Nature (London) 438, 201 (2005).

[28] P. A. Lee and T. V. Ramakrishnan, Rev. Mod. Phys. 57, 287 (1985).

[29] M. O. Goerbig, J.-N. Fuchs, G. Montambaux, and F. Piéchon, Phys. Rev. B 78, 045415 (2008).

[30] M. Plihal and A. A. Maradudin, Phys. Rev. B 44, 8565 (1991).

[31] O. Peleg, G. Bartal, B. Freedman, O. Manela, M. Segev, and D. N. Christodoulides, Phys. Rev. Lett. 98, 103901 (2007).

[32] L.-G. Wang, Z.-G. Wang, J.-X. Zhang, and S.-Y. Zhu, Opt. Lett. 34, 1510 (2009).

[33] X. Huang, Y. Lai, Z. H. Hang, H. Zheng, and C. T. Chan, Nat. Mater. 10, 582 (2011).

[34] L. Sun, J. Gao, and X. Yang, Opt. Express 21, 21542 (2013).

[35] M. Born, E. Wolf, and A. Bhatia, Principles of Optics: Electromagnetic Theory of Propagation, Interference and Diffraction of Light (Cambridge University, Cambridge, 1999).

[36] G. A. Niklasson, C. G. Granqvist, and O. Hunderi, Appl. Opt. 20, 26 (1981).

[37] L. Landau, E. Lifshitz, and L. Pitaevskii, Electrodynamics of Continuous Media (Pergamon, New York, 1984).

[38] E. Hecht, Optics (Addison-Wesley, Reading, MA, 2002).

[39] W. Gao, M. Lawrence, B. Yang, F. Liu, F. Fang, J. Li, and S. Zhang, arXiv:1401.5448.

[40] R. A. de Groot, F. M. Mueller, P. G. van Engen, and K. H. J. Buschow, Phys. Rev. Lett. 50, 2024 (1983).

[41] A. B. Khanikaev, S. Hossein Mousavi, W.-K. Tse, M. Kargarian, A. H. MacDonald, and G. Shvets, Nat. Mater. 12, 233 (2013).

[42] D. Dragoman and M. Dragoman, Quantum-Classical Analogies, The Frontiers Collection (Springer, New York, 2004).

[43] F. D. M. Haldane, Phys. Rev. Lett. 61, 2015 (1988).

[44] C. L. Kane and E. J. Mele, Phys. Rev. Lett. 95, 146802 (2005).

[45] M. Z. Hasan and C. L. Kane, Rev. Mod. Phys. 82, 3045 (2010).

[46] S. Raghu and F. D. M. Haldane, Phys. Rev. A 78, 033834 (2008).

[47] T. Ochiai and M. Onoda, Phys. Rev. B 80, 155103 (2009).

[48] M. C. Rechtsman, Y. Plotnik, J. M. Zeuner, D. Song, Z. Chen, A. Szameit, and M. Segev, Phys. Rev. Lett. 111, 103901 (2013).

[49] M. C. Rechtsman, J. M. Zeuner, Y. Plotnik, Y. Lumer, D. Podolsky, F. Dreisow, S. Nolte, M. Segev, and A. Szameit, Nature (London) 496, 196 (2013). 